\title{
Crosswise Recursive Algorithm of Optimized Wrinkling for Quadric Surface Metal Curtain Wall
}

\author{
Yalong Zhang ${ }^{* 1,3}$ and Xuan $\mathrm{Ma}^{2}$ \\ ${ }^{1}$ College of Electrical and Information Engineering, Quzhou University, Quzhou 324000, China \\ ${ }^{2}$ Faculty of Automation and Information Engineering, Xi' an University of Technology, Xi'an 710048, China \\ ${ }^{3}$ School of Electrical, Electronic and Computer Engineering, University of Western Australia, Perth 6009, Australia
}

\begin{abstract}
Metal curtain walls have been used to decorate the interiors and exteriors of large buildings. Most exteriors used in performing surface modeling of large buildings are linked or pieced by quadric surface. This surface, which is non-expansible, will cause wrinkling when stamped and used as a flat sheet metal of a metal curtain wall. These wrinklings are not distributed effectively because of the curvature asymmetry of surface modeling. Hence, this study proposes a horizontal recursive algorithm of optimized wrinkling that will enable effective distribution of wrinklings when the surface is stamped to form a shape, thereby satisfying the requirements of precision in surface stamping.
\end{abstract}

Keywords: Flat unfolding, metal curtain wall, optimized wrinkling, quadric surface.

\section{INTRODUCTION}

Metal curtain walls have been widely used to decorate exteriors of large buildings because of its advantages, such as providing protection for the building, anti-corrosion feature, noise-proof, and attractiveness. Creating a metal curtain wall with surface modeling requires a complex manufacturing techniques. First, a three-dimensional (3D) surface is divided into several smaller surface patches following certain attractiveness requirements. Each surface patch is then plasticized and stamped using a flat sheet metal. Among the commonly used basic surface forms include, paraboloid, ellipsoid, and sphere surfaces. Surfaces of complicated modeling are also linked or pieced mainly through the above-mentioned surfaces.

A quadric surface is non-expansible, and thus it may inevitably cause wrinkling when stamped from flat to surface. Conical and cylindrical surfaces can be formed from a flat surface by curving it without any wrinkling. Several studies [1-10] on both methods of shaping non-expansible surface have addressed certain problems in relevant projects of surface processing.

To create an attractive decoration for surface metal curtain wall, the junctures fitted along the rear surface patches of the exterior surface of the buildings should be distributed effectively. Satisfying this requirement depends on manufacturing techniques. The commonly used expansion algorithm [3] does not consider the requirement of equal apothems and thus is not suitable for use in surface metal curtain walls. The expansion algorithm of equal apothems

*Address correspondence to this author at the College of Electrical and Information Engineering, Quzhou University, Quzhou 324000, China; Tel: 86-0570-8015081; Fax: 86-0570-8015081;

E-mail: yalong-2008@hotmail.com
[11] considers the requirements of invariable distance from the boundary point to the center after surface flattening. However, the expansion algorithm fails to consider wrinkling distribution. Radial (longitudinal) wrinkling creates transverse wrinkling because of the different wrinkling densities when the surface is punch-formed. The boundary point drifts when dealing with the surface patch of curvature dissymmetry, which will affect the requirements of constructional shapes.

The transverse directions of the radial wrinkling are actually concentric circles with a variety of sizes. Surface flattening for an even distribution of wrinkling can be achieved by defining the wrinkling density and then relocating their transverse positions with even wrinkling density. The difficulty therefore lies in how to achieve the adjustment and compensation of the apothems after the transverse locations are moved. When moving to a given point, determining the new position to homogenize the wrinkling density is easy; however, determining which point will move to the present location can be difficult. In this case, both the transverse and longitudinal locations should be changed to guarantee evenness of the wrinkling density and ensure that the plane can be stamped accurately into the required surface.

Based on literature [11], this study proposes a horizontal recursive algorithm of boundary point to calculate the horizontal shifting measures produced by surface stamping to offer the horizontal position of boundary point when the wrinkling moves. Small changes in the lengthwise position can be adjusted through its horizontal position to satisfy the requirement of the entire shaping and ensure that the stamped and moved shape can satisfy the requirement of assembling shape. 


\section{CROSSWISE RECURSIVE ALGORITHM}

\subsection{Description of the Problem}

The corresponding flat shape by a given surface patch should be calculated to extend the surface curtain wall. This calculation will enable the surface of the flat shape to satisfy the requirements of surface assembly after punch-forming. These requirements include smooth and orderly junctures that eliminate the need for repeated repairs because of incorrect shapes in current manual models.

In calculating the corresponding flat shape of the surface, an approximate position of the surface patch should be confirmed as the corresponding center of the plane patch. The position of the boundary point on the flat shape should then be calculated relative to the center point to confirm the flat shape. This procedure is conducted to confirm the flat shape. Given the radial wrinkling lines, the boundary points are considered the terminal point of the wrinkling lines. The positions of the boundary points can be divided into lengthwise (radial) and crosswise directions. The lengthwise (radial) and crosswise directions are relative to the radial and crosswise directions of the wrinkling lines, instead of radial and crosswise directions in the rectangular coordinate system. The radial position refers to the distance between the boundary points to the center point. The radial position has been confirmed by literature [8]. However, this approach fails to consider that the boundary point requires optimization when in the crosswise direction. Hence, this study aims to solve the second problem, namely, the optimized position of boundary point in the crosswise direction.

\subsection{Crosswise and Lengthwise Preliminary Positions of the Boundary Point}

Determining the central point of a surface is comparatively easy, and can be achieved through an experiment where neither the near point nor the centrobaric near point of a geometric center is applied because the surface center has a considerable effect on calculation results. The computation method for the lengthwise boundary point and the confirmation prescription of the crosswise boundary point in the equal apothem expansion methods are introduced first [11], and then the crosswise recursive algorithm is introduced.

\subsubsection{Lengthwise Position Calculation}

Fig. (1) shows that surface $A B C D$ is the given surface patch and that the central point is $P$. Point $E$ should be one of the boundary points in the surface patches to enable normal $P Q$ to cross point $P$ and plane $P Q M E$ to cross $P Q$, which intersects with plane $A B C D$ in $P E^{-}$. The curve length of $P E^{-}$can be calculated by selecting point $E^{\prime}$ on the intersected line of normal plane $P Q$ and plane $P Q M E$, making straight-line distance $P E^{\prime}=P E^{-}$the corresponding point of point $E$ on the plane. Assuming $P Q$ as rotation plane $P Q M E, P Q$ should be intersected with a given surface boundary point. The curve length of the intersection line is then calculated. The curve length of the boundary point and central point $P$ is considered as the apothem on the plane that will enable the lengthwise positions of other boundary points on the plane to be obtained.
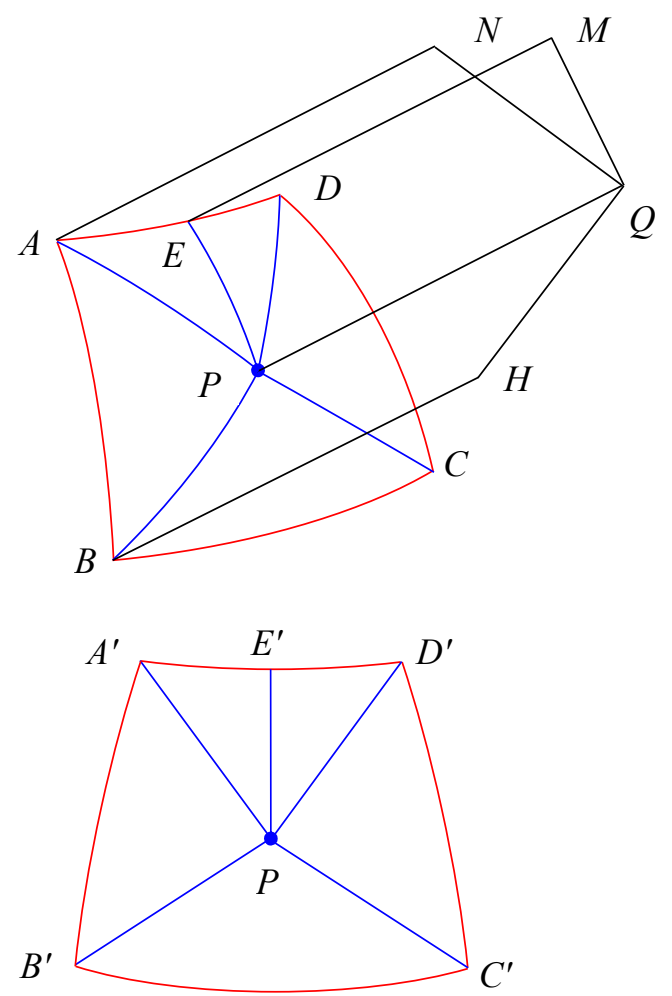

Fig. (1). Sketch map of the surface flattening.

Given that the surface equation of a given surface in a 3D space is $F(x, y, z)=0$, its partial derivative $\left(F_{x}, F_{y}, F_{z}\right)$ in the 3D coordinate system is the direction of the surface patch normal $P Q$. Considering that the coordinate of point $P$ has been provided, the following point normal form equation for the straight-line $P Q$ can be obtained:

$\frac{x-x_{p}}{\left.F_{x}\right|_{p}}=\frac{y-y_{p}}{\left.F_{y}\right|_{p}}=\frac{z-z_{p}}{\left.F_{z}\right|_{p}}$

Supposing point $E$ is $\left(x_{e}, y_{e}, z_{e}\right)$, the normal vector of plane $P Q M E$ is as follows:

$\left(\left.F_{x}\right|_{p},\left.F_{y}\right|_{p},\left.F_{z}\right|_{p}\right) \times\left(x_{e}-x_{p}, y_{e}-y_{p}, z_{e}-z_{p}\right)$

$=\left[\begin{array}{ccc}i & j & k \\ \left.F_{x}\right|_{p} & \left.F_{y}\right|_{p} & \left.F_{z}\right|_{p} \\ x_{e}-x_{p} & y_{e}-y_{p} & z_{e}-z_{p}\end{array}\right]$

The point normal form equation of plane $P Q M E$ is as follows:

$\left[\left.\left(y_{e}-y_{p}\right) F_{z}\right|_{p}-\left.\left(z_{e}-z_{p}\right) F_{y}\right|_{p}\right]\left(x-x_{p}\right)$
$+\left[\left.\left(z_{e}-z_{p}\right) F_{x}\right|_{p}-\left.\left(x_{e}-x_{p}\right) F_{z}\right|_{p}\right]\left(y-y_{p}\right)$
$+\left[\left.\left(x_{e}-x_{p}\right) F_{y}\right|_{p}-\left.\left(y_{e}-y_{p}\right) F_{x}\right|_{p}\right]\left(z-z_{p}\right)$
$=0$

Curve $P E^{-}$can be obtained immediately when Eq. (3) is connected with the surface equation $F(x, y, z)=0$. The length of $P E^{-}$can be regarded as the plane length between point $E$ 
and central point $P$ on the plane after extension of the surface, and is called the lengthwise position.

The positions of all boundary points on the plane can be determined by assuming that the boundary point step size is a fixed value. Diagram 1 shows that plane $P Q N A$ intersects with curve $P A^{-}$, and the length of curve $P A^{-}, P A^{\prime}$, can determine the lengthwise position of point $A$ on the unfolded plane. The rest of the positions can be determined in a similar manner. Points $B, C$, and $D$ can also be located. The unfolded plane $A^{\prime} B^{\prime} C^{\prime} D^{\prime}$ of surface $A B C D$ is then formed.

\subsubsection{Preliminary Calculation of Crosswise Position}

Suppose the normal vectors of two planes are $\left(a_{1}, b_{1}, c_{1}\right)$ and $\left(a_{2}, b_{2}, c_{2}\right)$, their dihedral angles can be obtained using the following formula:

$\cos \phi=\frac{a_{1} a_{2}+b_{1} b_{2}+c_{1} c_{2}}{\sqrt{a_{1}^{2}+b_{1}^{2}+c_{1}^{2}} \cdot \sqrt{a_{2}^{2}+b_{2}^{2}+c_{2}^{2}}}$

Considering point $E$ as standard, the dihedral angle derived by Eq. (4) can be considered as the intersection angle between each boundary point and point $E$ on the unfolded plane, similar to the intersection angles between $\angle E P A^{\prime}$, $\angle E P B^{\prime}, \angle E P C^{\prime}$, and so on. Datum point $E$ ascertains the preliminary crosswise positions of points $A^{\prime}, B^{\prime}$, and $C^{\prime}$ on the plane in Fig. (1).

The curve length of the intersecting surface and the plane can be calculated during the unfolding process. However, similar to the lengths of $P E^{-}$and $P A^{-}$, the calculation can be rather complicated because of the lack of commonly used mathematical formulas. Hence, the method for curve segmentation fitting can be employed to solve this problem. The curve can be divided into several smaller sections by replacing each section with a parabolic length that crosses both end and midpoint. The fitting length of all of sections is the curve length.

\subsection{Crosswise Recursive Algorithm of Boundary Points}

The unfolding process determines the horizontal positions of the boundary points on the plane. However, the process fails to consider the crosswise shifting of the boundary points after homogenization of wrinklings. Hence, after obtaining the measurement of crosswise shifting, the lengthwise length can be adjusted properly based on the apothem requirement of assembling surface. The measurement also satisfies the requirements of surface assembly shapes. The adjustment of the lengthwise length is not discussed in this paper as it is not the focus of the study. The calculation method for crosswise shifting measures for boundary points can be found below.

Fig. (2) shows that given surface patch $A B C D$, the crosswise recursive algorithm of boundary point $E$ can e considered as an example, and that the calculations of other boundary points merely follow this pattern.

Suppose the exterior curve length of the surface patch $P E^{-}$is $R_{1}$ and the vertical distance from point $E$ to space normal $P Q$ is $R_{2}$, therefore, the wrinkling density coefficient of point $E$ is defined as $1-R_{2} / R_{1}$. This result indicates several points, including that the curvature of curve $P E^{-}$is larger, the distance from point $E$ to space normal $P Q$ is shorter, and the wrinkling density of point $E$ is also larger. The point of the large wrinkling density also shifts toward to the point of the small wrinkling density because of the malleability of the sheet metal. Similarly, the wrinkling density coefficient of an arbitrary point on the surface is defined as,

$W_{X}=1-P X / \overparen{P X}$

where $P X$ is the distance from point $X$ to space normal $P Q$ and $P X^{-}$curve length of the surface from point $P$ to point $X$.

A quasi-circle $C_{P}$ can be drawn by considering point $P$ as the center and the length of curve as the radius. This circle can be called the quasi-circle because it is not a proper circle and their distances from each point on the circumference to central point $\mathrm{P}$ are equal instead of equal straight-line distances. Therefore, the circles may not be on the same plane, similar to the dashed circumference in Fig. (2).

Take point $A$ as an example; to create a plane across normal $P Q$ of point $P$, a crossing plane $P Q N A$ of point $A$ should intersect with the surface at curve $P A^{-} . P A^{-}$and $C_{P}$ intersect at point $A_{0}$. With the apothem of point $A$ unchanging, the new direction angle of point $\mathrm{A}$ is re-located using the following:

$\theta_{A}^{\prime}=\theta_{A}-\Delta \theta$

where

$$
\begin{aligned}
& \Delta \theta=2 \pi\left[\int_{0}^{\theta_{A}} W_{C_{P}(\theta)} d(\theta) / \int_{0}^{2 \pi} W_{C_{P}(\theta)} d(\theta)\right]-\theta_{A} \\
& \therefore \quad \theta_{A}^{\prime}=2 \theta_{A}-2 \pi\left[\int_{0}^{\theta_{A}} W_{C_{P}(\theta)} d(\theta) / \int_{0}^{2 \pi} W_{C_{P}(\theta)} d(\theta)\right]
\end{aligned}
$$

where $C_{P}(\theta)$ is the point which belongs to $C_{P}$ and whose direction angle is $\theta . W_{C p(\theta)}$ is the wrinkling density coefficient of point $C_{P}(\theta)$. We can see from Eq.(6) that point $A$ moves towards the area with a larger wrinkling density. This movement is because only the movement toward the opposite direction has compensation effect. The example refers point $A$; the calculation of movement of the other boundary points can be performed in the same manner.

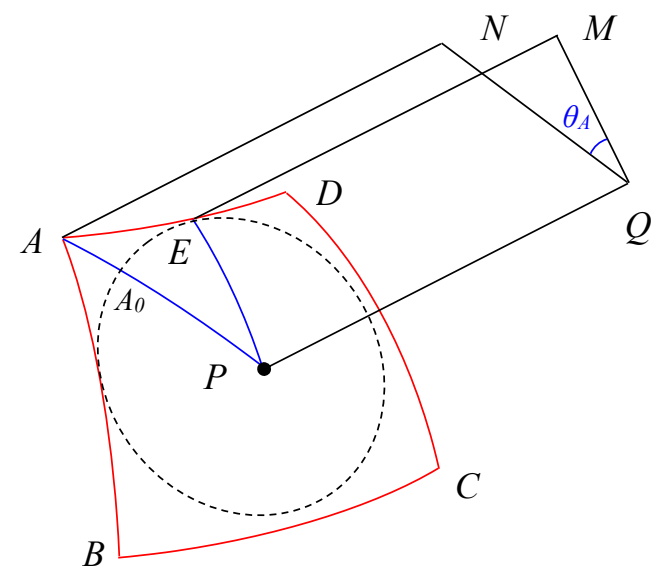

Fig. (2). Sketch map of crosswise recursive algorithm. 


\section{CALCULATION EXAMPLES}

The proposed algorithm was developed as an application system for use in the stamping process of surface curtain wall. Fig. (3) shows the interface of the application system that can handle a paraboloid with an equation of

$\frac{x^{2}}{a^{2}}+\frac{y^{2}}{b^{2}}=-2 p(z-h)$,

where $a=100, b=150, p=0.01$, and $h=200$. Four sections of the surface are selected and marked as 1,2, 3, and four. These sections are represented as shaded areas in the figure. The parameters of the four sections of the surface can be found in Table 1. The unfolded picture of surface patch 1-4 is similar to that in Fig. (4).

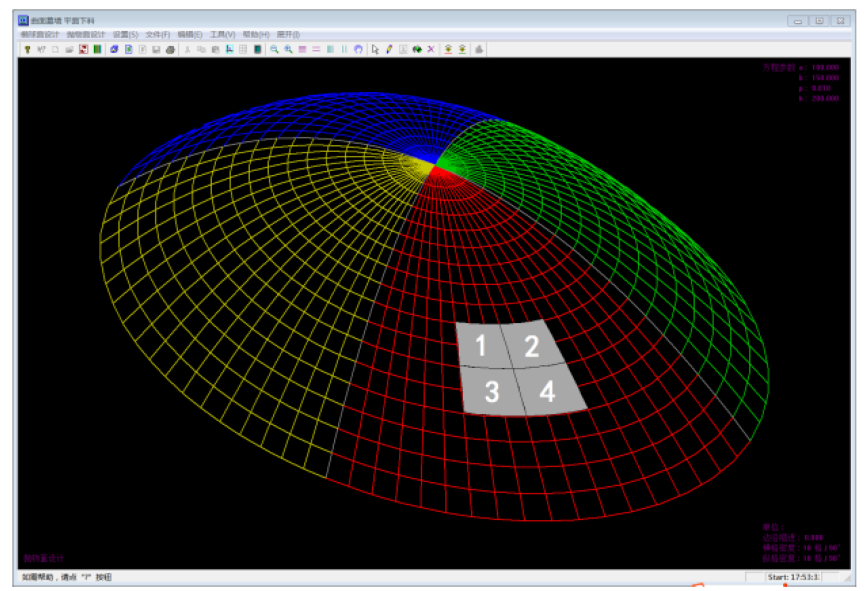

Fig. (3). Surface patch of paraboloid.

In Fig. (4), upper line shows the unfolded wrinkling distribution picture of surface patch 1-4 from left to right using the algorithm of equal apothems. The lower line is created using the proposed algorithm. The wrinkling indicates the wrinkling density rather than their specific location. We can learn from Fig. (4) that (i) the wrinkling density of four corners in each section surface created using the algorithm of equal apothems is larger than anywhere else, and the bigger the piece the more apparent it is; (ii) the wrinkling density created by the proposed algorithm is even along with the transverse directions of wrinkling; and (iii) the wrinkling density along with the longitudinal directions is determined by the curvature of surface instead of proposed algorithm. After crosswise shifting, the wrinklings are distributed effectively, making it more convenient to punchform the surface patches. The patches can be punched directly and formed into the necessary shapes and sizes after natural extension.

Table 1. Parameters of surface sections.

\begin{tabular}{|c|c|c|c|c|}
\hline No. & $\begin{array}{c}\text { Left } \\
\text { Longitude }\end{array}$ & $\begin{array}{c}\text { Right } \\
\text { Longitude }\end{array}$ & $\begin{array}{c}\text { Lower } \\
\text { Height }\end{array}$ & $\begin{array}{c}\text { Upper } \\
\text { Height }\end{array}$ \\
\hline \hline 1 & 35.592 & 51.258 & 111.655 & 139.575 \\
\hline 2 & 51.258 & 65.197 & 111.655 & 139.575 \\
\hline 3 & 35.592 & 51.258 & 79.156 & 111.655 \\
\hline 4 & 51.258 & 65.197 & 79.156 & 111.655 \\
\hline
\end{tabular}

The plane unfolded shape of surface 1-4, as illustrated by the algorithm of equal apothems in upper line of Fig. (4), appears unchangeable with the 3D vision of the surface patch because of the comparatively small size of the selected surface patches as compared with the entire surface. Thus, the curvature is not obvious. However, the computational accuracy of a 64-bit computer is extremely high and can satisfy the requirement for precise reserved distances among junctures when fitting the rigid metal plate with proposed

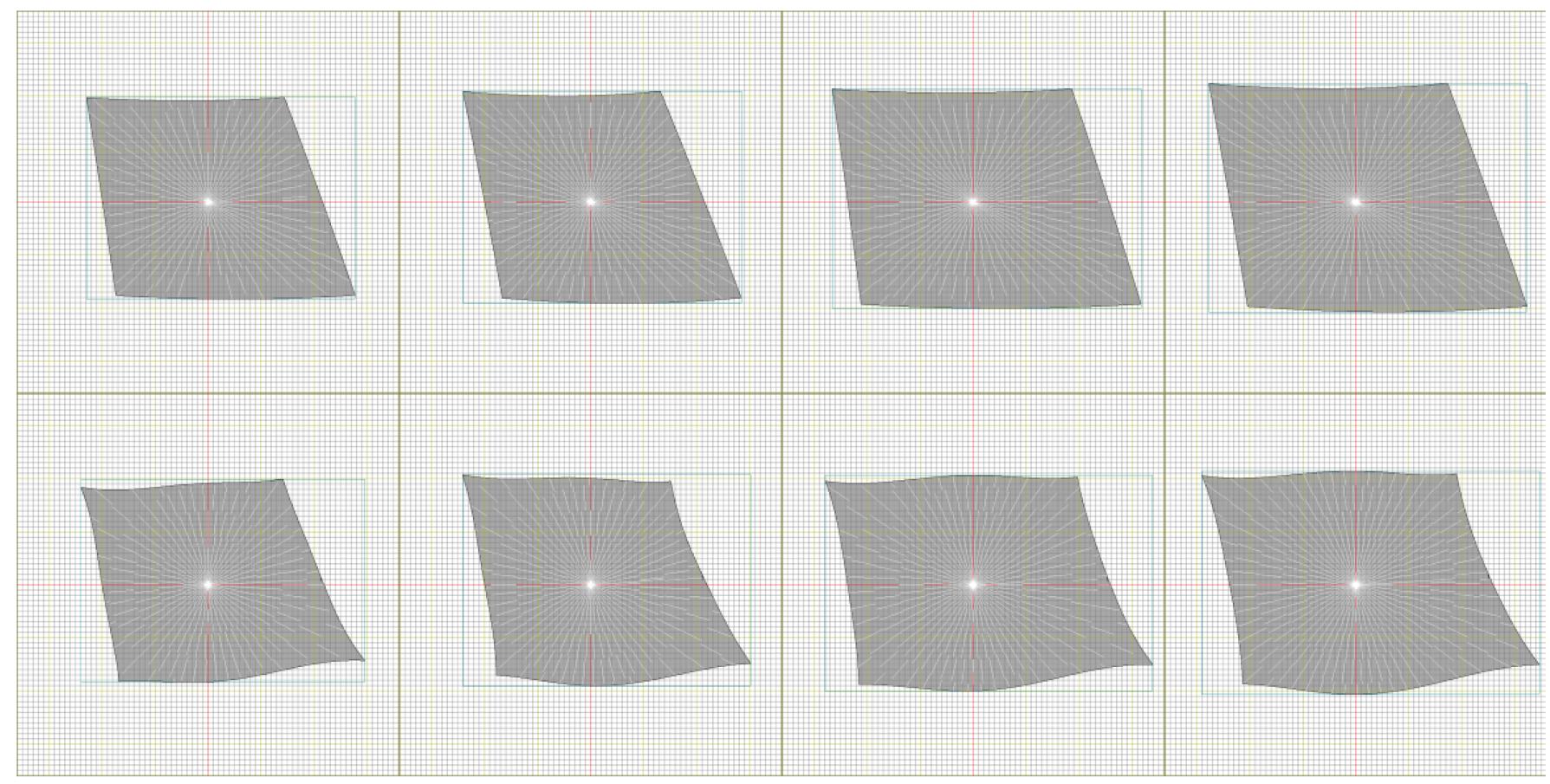

Fig. (4). Unfolded picture and contrast pictures of wrinkling optimization. 
algorithm. The precise reserved distances enable the effect of beauty to be achieved. In practical projects, distribution of surface patches may either be larger of smaller, with the curvature being more obvious when it is larger. The distinction between results and surface $3 \mathrm{D}$ profile is also larger.

\section{CONCLUSION}

The unfolded crosswise recursive algorithm of a quadric surface curtain wall on the plane determines the deformation of wrinklings from the direction of smaller density to that of the larger density when punching sheet metal. The algorithm also offers a calculation method of shifting measurements to effective distribution of the wrinklings when punching the surface into shape. The method simplifies the requirements of the punching technique. Combined with the expansion method of the equal apothem [11], a crosswise recursive algorithm was developed into a computer application system. The developed computer application system exhibited effectivity in practical production. The model replaces the previous manual model and largely enhances production efficiency. The model also simplifies sheet metal forming technology through refined calculations, resulting in significant improvement in the quality of products.

\section{CONFLICT OF INTEREST}

The authors confirm that this article content has no conflict of interest.

\section{ACKNOWLEDGEMENTS}

This study was subsidized by the scientific research start expenditure of Quzhou University (Item No: BSYJ201309) as well as by the guiding science and technology planning project of Quzhou Municipal Science and Technology Bureau (Item No: 2013048).

\section{REFERENCES}

[1] Q. Hong, X. Huang, L. G. Li, and Y. D. Bao, "Research and implementation of metal plate's unfoldable surface flattening finite element backflush", Machine Manufacturing \& Automation, vol. 40, pp. 37-39, February 2011.

[2] Y. P. Xue, and Z. W. Chen, "Applied research of rhino in the manufacture of surface metal curtain wall plate", Machine Design \& Manufacturing Engineering, vol. 43, pp. 67-70, April 2014.

[3] Y. B. Liang, W. C. Xu, J. G. Li, and Q. Yang, "Surface flattening universal algorithm based on mechanical model", Computer Engineering \& Designing, vol. 33, pp. 3539-3543, September 2012.

[4] G. B. Yan, and B. Liu, "A surface flattening improved algorithm based on energy model", Journal of Huaqiao University (Natural Science Edition), vol. 32, pp. 135-139, February 2011.

[5] B. Yang, J. P. Chen, L. Yang, and L. Z. Lai, "Applications in wing wallboard unfolding \& modeling of reverse engineering and secondary development technology", CAD/CAM and Information of Manufacturing Industry, vol. 9, pp. 49-53, September 2014.

[6] X. K. Cui, and M. Chen, "Topology optimal unfoldable gridding surface lofting modeling", Computer Application, vol. 32, pp. 2798-2801, 2813, October 2012.

[7] X. K. Cui, and M. Chen, "Curve design of quickly unfoldable narrow-band", Applied Research of Computer, vol. 29, pp. 39974000, October 2012.

[8] J. Bin, Z. Minghui, W. Zhigang, and G. Yancong, "Model analysis on hardened steel surface topography characteristic in high speed milling", The Open Mechanical Engineering Journal, vol. 9, pp. 219-255, September 2015.

[9] J. Wei, and S. Song, "Residual static strength of tubular t-joints with fatigue surface cracks", Open Mechanical Engineering Journal, vol. 9, pp. 1-6, September 2015.

[10] Y. Haiyang, W. Yunxin and L. Zhiqi, "A damage tolerance model for plates with surface cracks under combined end force and bending", Open Mechanical Engineering Journal, vol. 8, pp. 562566, August 2014.

[11] X. Ma, Y. L. Zhang, and S. M. Li, "Equal apothem unfolding method of double curved surface metal curtain wall", Computer Engineering and Application, vol. 45, pp. 217-218, 235, November 2009. 